\title{
Study on Transport of Molecules in Gel by Surface- Enhanced Raman Spectroscopy
}

\section{Samir Kumar ( $\nabla$ samiratwork@gmail.com )}

㽖 / Kyoto University https://orcid.org/0000-0002-6302-6326

\section{Taneichi Taiga}

Kyoto University: Kyoto Daigaku

\section{Takao Fukuoka}

Kyoto University: Kyoto Daigaku

\section{Kyoko Namura}

Kyoto University: Kyoto Daigaku

\section{Motofumi Suzuki}

Kyoto University: Kyoto Daigaku

\section{Research Article}

Keywords: Cellulose, SERS, Diffusion, Au nanorods

Posted Date: July 22nd, 2021

DOI: https://doi.org/10.21203/rs.3.rs-677741/v2

License: (c) (i) This work is licensed under a Creative Commons Attribution 4.0 International License. Read Full License

Version of Record: A version of this preprint was published at Cellulose on October 12th, 2021. See the published version at https://doi.org/10.1007/s10570-021-04249-z. 


\section{Study on Transport of Molecules in Gel by Surface-Enhanced Raman Spectroscopy}

3 Department of Micro Engineering, Graduate School of Engineering, Kyoto University, Katsura, Nishikyo, Kyoto,

$$
\text { 615-8540, Japan }
$$

\section{Corresponding Author}

$6 \quad *$ SK: drsamirkumar2017@gmail.com, MS: m-snki@me.kyoto-u.ac.jp

\section{ABSTRACT}

8 Surface-enhanced Raman spectroscopy (SERS)-based biosensors have recently been extensively developed because

9 of their high sensitivity and non-destructive nature. Conventional SERS substrates are unsuitable for detecting 10 biomolecules directly from human skin. As a result, significant effort is being put into developing a gel-type SERS 11 sensor that can segregate and detect biomolecules due to differences in molecular transport phenomena in the gel.

12 However, no comprehensive research studies on the transport processes of molecules in gels in gel-type SERS sensors

13 have been reported. This paper reports the differences in the transport phenomena of different molecules based on the 14 time change of SERS spectrum intensity. The Au nanorod array substrate was coated with HEC gel to prepare a sample 15 cell to study diffusion. The SERS spectra of aqueous solutions of 9 types of molecules were measured using the 16 prepared sample cells. The rate at which each molecule diffuses into the gel differs depending on the molecule. The

17 time variation of the characteristic SERS peak of each molecule was investigated based on a one-dimensional diffusion

18 model, and the diffusion coefficient $D$ was calculated for each molecule. A comparative study was conducted on the 19 relationship between the diffusion coefficient and the molecular weight and molecular size, and it was found that the

20 larger the molecular weight and molecular size, the slower the diffusion, which is based on the molecular motion 21 theory and the inhibitory effect of the gel substance.

\section{Keywords: Cellulose, SERS, Diffusion, Au nanorods}

\section{1. INTRODUCTION}

24 Surface-enhanced Raman spectroscopy (SERS) is a vibrational spectroscopic technique that has emerged as a 25 promising method for the non-destructive study of materials down to the single-molecule level.(Schlucker 2013) 
26 SERS has proven to be a powerful analysis tool for molecular structure analysis, cell imaging, and biomolecule 27 detection, among other things.(Kumar et al. 2015; El-Zahry and Lendl 2018; Yu et al. 2020; Hickey and He 2021)

28 SERS has found its application not only in the fields of physics, chemistry, and biology, but also in engineering,

29 pharmacy, and medicine.(McNay et al. 2011; Sharma et al. 2012; Bochenkov et al. 2015; Singh et al. 2019; Segawa

30 et al. 2019a; Kumar et al. 2020c, d) Surface-enhanced Raman scattering is the phenomenon of enormous enhancement

31 in the Raman scattering cross-section of molecules adsorbed in the vicinity of plasmonic nanoparticles.(Le Ru and

32 Etchegoin 2009) Recently, SERS-based biosensors have been proposed for the detection of trace levels of 33 biomolecules and diagnostics.(Kumar et al. 2015; Premasiri et al. 2018; Joseph et al. 2018) A SERS substrate is any

34 nanostructure that supports SERS enhancement. Conventional SERS substrates are metal nanoparticles in either a 35 colloidal solution or a solid substrate.(Suzuki et al. 2006; Rajput et al. 2017; Segawa et al. 2019b; Gahlaut et al. 2020;

36 Yadav et al. 2021) As the colloidal solution is liquid and the structure of the solid substrate is rigid and brittle, it

37 drastically limits the applicability of the SERS sensor and makes it unsuitable for detecting biomolecules directly from

38 the human skin. As a result, a new gel-type porous SERS sensor that can collect biomarkers directly from the surface

39 should be developed.

$40 \mathrm{Yu}$ and White have reported paper-based SERS devices for chromatographic separation and detection of target 41 analytes in complex samples.(Yu and White 2013) Similarly, because of differences in the transport processes of 42 biomolecules that permeate through the gel, gel-based SERS sensors may differentiate and detect biomolecules. If this 43 technology is developed, it may serve as a biosensor and a new means of analyzing biomolecules. Recently, we 44 reported a gel-based SERS sensor for the direct collection of biomarkers from the skin.(Kumar et al. 2019, 2020b) We 45 found that the probe molecule solution permeated through the gel quickly and could be detected by SERS within 1 46 minute. However, no studies have been reported on the time-dependent SERS spectrum for the transport phenomena 47 of various molecules and differences in transport phenomena in gels depending on the type of molecule. A few other 48 reports on the gel-based SERS sensor and the diffusion studies of molecules in a gel.(Lauffer 1961; Muhr and 49 Blanshard 1982; Amsden 1998; Samprovalaki et al. 2012; Tokita 2016; Sandrin et al. 2016; Chen et al. 2019a, b, 50 2021; Innocenzi and Malfatti 2019; Ogundare and van Zyl 2019; Wu et al. 2020; Kim et al. 2020; Hu et al. 2021)

51 However, the focus of these studies was on the SERS enhancement and practical application of this sensor and 52 theoretical studies on the diffusion of micro solutes in a homogeneous gel. To our knowledge, the transport phenomena 53 of molecules with varying molecular weight in the gel have not been elucidated using SERS. 
54 The diffusion of molecules is essential not only from a fundamental physics standpoint, but also for adequately 55 evaluating the diffusion of drugs and particles,(Lock et al. 2018) transport of molecules in tumors,(Jain 1987) and 56 release of small bioactive molecules from physical gels for the encapsulation and controlled release of small

57 therapeutic molecules(Mayr et al. 2018). This study aims to elucidate the difference in transport phenomena of 58 different types of molecules based on SERS spectral intensity time change. The time change of the SERS spectra of 59 an aqueous solution of nine different molecules was studied using a Hydroxyethylcellulose (HEC) gel-based cell. A 60 method for calculating the SERS spectral intensity from collected data and characterizing the transport of molecules

61 in the gel was also developed. Finally, the temporal change in SERS spectral intensity was fitted using the model. The 62 diffusion phenomena were quantified for nine types of molecules, and the relationship between molecular weight and 63 molecular size and the diffusion phenomena was investigated.

\section{MATERIALS AND METHOD}

\section{$65 \quad 2.1$ Gel and Raman probe molecules.}

66 HEC gel, which is non-toxic to humans and is used as a thickening in cosmetics and external medicines, was used 67 to prepare the gel-based cell for the experiment. HEC is a polymer compound in which a hydroxyl group is added to 68 cellulose to make it water-soluble, and when mixed with water, it behaves as a transparent gel. The feasibility of a 69 gel-based SERS sensor using HEC gel has been reported by Kumar et al.(Kumar et al. 2020b) HEC powder (SE400, 70 Daicel FineChem Ltd.) was mixed with ultrapure water to a mass ratio of 10\%, then well mixed and allowed to stand 71 overnight or more to form an HEC gel from which bubbles were removed.

72 The molecular weight, molecular formula, van der Waals volume (vdW volume), and solvent-exposed surface area 73 (SEA) of the nine types of Raman probes used in this study are shown in Table 1, and the molecular structure is shown

74 in Figure S1 (Supplementary Information). The vdW volume of a molecule is defined as the space occupied by the 75 molecule inaccessible to other molecules at room temperature(Askadskii 2003), and SEA is defined as the surface 76 area of a molecule in which the molecule can come into contact with the solvent.(Hamelryck 2005)

77 Table 1. Molecular weight, molecular formula, van der Waals volume, solvent exposure area of the molecule 78 used for measurement. 


\begin{tabular}{lllll}
\hline Molecule name & $\begin{array}{l}\text { Molecular } \\
\text { weight }\end{array}$ & Molecular formula & $\begin{array}{l}\text { vdW volume } \\
{\left[\AA^{3}\right]}\end{array}$ & $\begin{array}{l}\text { Solvent } \\
\text { exposure area } \\
{\left[\AA^{2}\right]}\end{array}$ \\
\hline Pyridine & 79.10 & $\mathrm{C}_{5} \mathrm{H}_{5} \mathrm{~N}$ & 77.82 & 241.92 \\
\hline $4,4^{\prime}$-Bipyridine & 156.18 & $\mathrm{C}_{10} \mathrm{H}_{8} \mathrm{~N}_{8}$ & 144.04 & 329.57 \\
\hline 4,4'-Vinylenedipyridine & 182.22 & $\mathrm{C}_{12} \mathrm{H}_{10} \mathrm{~N}_{2}$ & 170.81 & 379.92 \\
\hline (S)-Equol & 242.27 & $\mathrm{C}_{15} \mathrm{H}_{14} \mathrm{O}_{3}$ & 218.54 & 413.02 \\
\hline Acid Orange 7 & 350.32 & $\mathrm{C}_{16} \mathrm{H}_{11} \mathrm{~N}_{2} \mathrm{NaO}_{4} \mathrm{~S}$ & 263.99 & 548.08 \\
\hline Acid Orange 12 & 350.32 & $\mathrm{C}_{16} \mathrm{H}_{11} \mathrm{~N}_{2} \mathrm{NaO}_{4} \mathrm{~S}$ & 262.44 & 517.84 \\
\hline Crystal Violet & 407.99 & $\mathrm{C}_{25} \mathrm{H}_{30} \mathrm{ClN}_{3}$ & 418.21 & 823.03 \\
\hline Rhodamine 6G & 479.02 & $\mathrm{C}_{28} \mathrm{H}_{31} \mathrm{ClN}_{2} \mathrm{O}_{3}$ & 377.39 & 765.18 \\
\hline Rose Bengal & 1049.85 & $\mathrm{C}_{20} \mathrm{H}_{2} \mathrm{Cl}_{4} \mathrm{I}_{4} \mathrm{~K}_{2} \mathrm{O}_{5}$ & 420.68 & 610.65 \\
\hline
\end{tabular}

\subsection{Preparation of diffusion cells}

80 The prepared sample cell is shown in Fig. 1. A $1 \mathrm{~mm}$ thick silicone rubber was sliced into $20 \mathrm{~mm} \times 25 \mathrm{~mm}$ pieces,

81 with a $12 \mathrm{~mm} \times 12 \mathrm{~mm}$ hole in the center. The prepared rubber was placed on a cover glass measuring $22 \mathrm{~mm} \times 26$

$82 \mathrm{~mm}$. A small amount of HEC gel was applied to the cover glass to prevent bubbles from forming between the SERS

83 substrate and the cover glass, and then the SERS substrate was placed in the center and squeezed from above with

84 tweezers. On the SERS substrate, HEC gel greater than the volume of the rubber hole was applied. The excessively

85 applied HEC gel was horizontally scraped off from the surface of the rubber in the same manner as the doctor's blade

86 technique to prepare an HEC gel layer with a constant film thickness.

87 On top of that, another piece of rubber was applied in an overlapping pattern. To account for the surface drying of the

88 HEC gel over time, the sample cell was created immediately before each molecule measurement. The SERS spectrum

89 was monitored after $100 \mu \mathrm{L}$ of an aqueous solution of each Raman probe molecule was dropped from the top of the

90 gel and covered with a cover glass.

\section{$91 \quad 2.3$ Time-dependent SERS measurement and SERS chip}

92 SERS spectra were acquired using a Raman spectrometer (RAM200S; LambdaVision Inc). A 785-nm laser with a

$9350 \times$ objective and approximately $30-\mathrm{mW}$ power on the sample was used for excitation. $100-\mu \mathrm{L}$ droplets of $1-\mathrm{mM}$

94 aqueous solution of Raman probe molecules were deposited on the cell, and its SERS spectra were recorded as a

95 function of time. Data was collected every 30 seconds for a total of 1800 seconds. After each acquisition, the sample 
stage was shifted $50 \mu \mathrm{m}$ to minimize the effect on the SERS spectrum caused by sample deterioration caused by

97 repeated laser light irradiation of one place on the SERS substrate. Pyridine (PY), 4,4'-Bipyridine (BPY), 4,4'-

98 Vinylenedipyridine (BPE), Crystal Violet (CV), Rhodamine 6G (Rh6G), and Rose Bengal (RB) have $1 \mathrm{~s}$ exposure

99 duration and four accumulations. Since the SERS spectrum of (S)-Equol (SE), Acid Orange 7 (AO7), and Acid Orange

10012 (AO12) is weaker than that of other molecules, the exposure time was kept at 5 s. OriginPro 2018 (OriginLab

101 Corporation, Northampton, MA, USA) was used to process the SERS spectra and analyze the data. Before analysis,

102 all data were baseline adjusted. A fourth-order polynomial was fitted to the raw SERS spectra and subtracted for

103 baseline correction. For the diffusion analysis, the most intense peak area was selected.

(a)

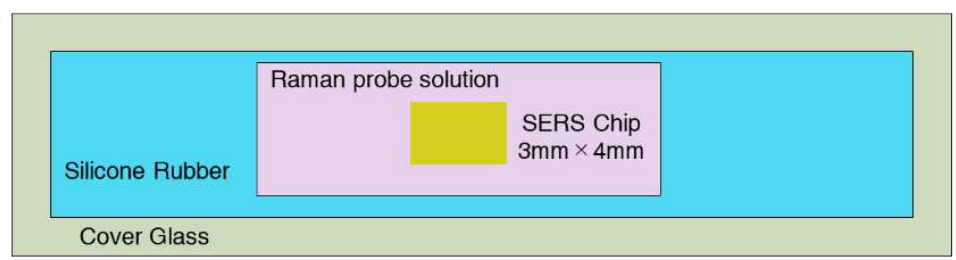

(b)
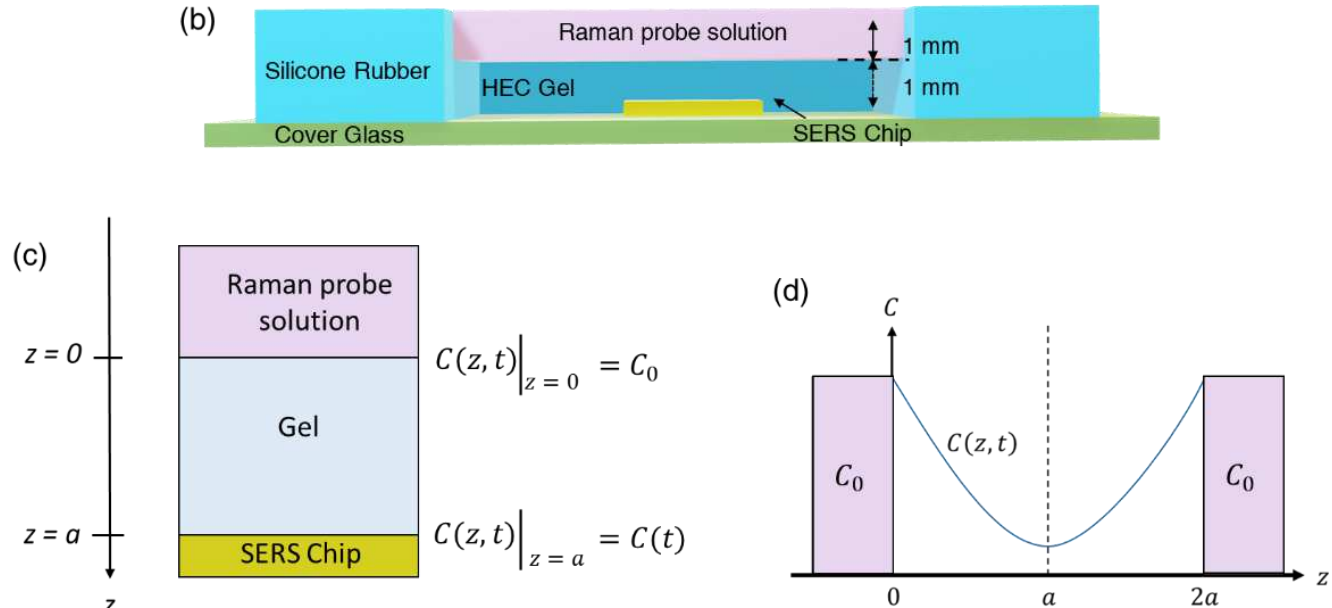

Fig. 1 Schematic diagram of diffusion cell (a) top view (b) cross-sectional view; Schematic diagram of crosssection of diffusion cell with boundary conditions; and (d) schematic diagram for the mirror image method to solve the diffusion equation.

104 The SERS chip was purchased from Nidek Co., Ltd, Japan. The SERS chip with elongated Au nanorod arrays

105 (AuNRAs) was developed by Suzuki et al. and is now commercially available as the Wavelet (Supplementary

106 information). The SERS chip was fabricated using a dynamic OAD technique.(Suzuki et al. 2005; Kumar et al. 2014,

107 2020a) The detailed fabrication process of AuNRAs can be found elsewhere.(Suzuki et al. 2007; Kumar et al. 2020e) 


\section{RESULTS AND DISCUSSION}

\section{$109 \quad 3.1$ Model for diffusion in gel}

110 In this section, we will examine the method to evaluate the intensity of the measured SERS spectrum. We will evaluate

111 a function that indicates the time change of the SERS spectral intensity. We will consider the diffusion of solutes in

112 homogeneous solvents on a macro scale using the diffusion equation for simplicity. According to Fick's first law, a

113 solute's diffusion flux is proportional to its concentration gradient. At this time, if the concentration is $C(z, t)$ as a

114 function of the coordinates $z$ in the vertical direction and the time $t$, and the amount of solute transported through the

115 unit area in the unit time is $J$, then

116

$$
J=-D \frac{\partial C(z, t)}{\partial z}
$$

117 Where $D$ is the diffusion coefficient of the solute molecule. Simultaneously, the mass flowing into the region of height

$118 z$ and $z+\Delta z$ during time $\Delta t$ is expressed as

$$
J(z) \Delta t-J(z+\Delta z) \Delta t=-\frac{\partial J}{\partial z}
$$

120 Since this is equal to the increase in solute concentration $\Delta \mathrm{C}(\mathrm{z}, \mathrm{t})$ during the time $\Delta t$, the following equation satisfies

121 the continuity equation.

$$
\frac{\partial C(z, t)}{\partial t}+\frac{\partial J}{\partial z}=0
$$

123 The following equation is obtained from equations (1) and (2).

$$
\frac{\partial C(z, t)}{\partial t}=D \frac{\partial^{2} C(z, t)}{\partial z^{2}}
$$

125 Equation 4 is called the diffusion equation. From kinetic theory, it is known that the diffusion coefficient is

126 proportional to the inverse square root of the molecular weight. The higher the diffusion coefficient, the faster the

127 diffusion. The transport processes of solutes can be described by providing the diffusion equation initial and boundary 128 conditions, and the diffusion coefficient $D$ can be used to quantify the diffusion of solutes. The diffusion equation of 129 Eq. (4) is modeled in one dimension for the sample cell as shown in Fig. 1(c), with the initial condition.

$$
\left.C(z, 0)\right|_{0<z \leq a}=0
$$

131 and boundary condition 


$$
\left.\frac{\partial C(z, t)}{\partial z}\right|_{z=a}=0
$$

134 As the molecules of interest are confined to a limited region and must satisfy the above boundary conditions in this 135 region, the schematic diagram in Figure 1(c) was replaced with Figure 1(d) using the mirror image method. The 136 solution of Eqn. 4 using the boundary conditions is given by

$$
C(z, t)=C_{0}\left\{1-\frac{4}{\pi} \sum_{n=0}^{\infty} \frac{1}{2 n+1} \cdot \exp \left[-\left\{\frac{(2 n+1) \pi}{2 a}\right\}^{2} D t\right] \cdot \sin \left[\frac{(2 n+1) \pi}{2 a} z\right]\right\}
$$

138 where a is the thickness of the gel layer. The detailed calculation can be found in the supplementary information.

139 By putting $z=a$ in the above equation, we get

$$
C(a, t)=C(t)=C_{0}\left\{1-\frac{4}{\pi} \sum_{n=0}^{\infty} \frac{(-1)^{n}}{2 n+1} \cdot \exp \left[-\left\{\frac{(2 n+1) \pi}{2 a}\right\}^{2} D t\right]\right\}
$$

141 Since the SERS spectral intensity of a molecule is proportional to its concentration,(Salemmilani et al. 2019; Wang et 142 al. 2019) the SERS spectral intensity $S(t)$ can be written as

$$
S(t)=S_{0}\left\{1-\frac{4}{\pi} \sum_{n=0}^{\infty} \frac{(-1)^{n}}{2 n+1} \cdot \exp \left[-\left\{\frac{(2 n+1) \pi}{2 a}\right\}^{2} D^{\prime}\right]\right\}
$$

144 Equation 7 will be used for fitting the SERS intensity curve in the linear region. When the solute diffuses into the 145 gel, it is considered to have three main diffusion inhibitory effects.(Lauffer 1961) The first is the interference of the 146 gel with the diffusion of solutes due to the obstruction effect and increased hydrodynamic drag. The second is that the

147 gel network may be thinner than the solute particles, and the third way the gel substance may affect the diffusion is 148 by binding the solute.(Muhr and Blanshard 1982) These effects are accounted for in the diffusion coefficient $D$ 149 reported from the experiments in this study.

\section{$150 \quad$ 3.2 SERS measurement}

151 First, let us consider the SERS spectrum of 4,4'-Bipyridine (BPY). BPY was chosen as the probe molecule because of

152 its well-established vibrational bands and lack of fluorescence.(Joo 2004) Fig. 2(a) shows the background and SERS

153 spectrum after $1800 \mathrm{~s}$ when the Raman peak was well stabilized. The four prominent characteristic Raman bands of 

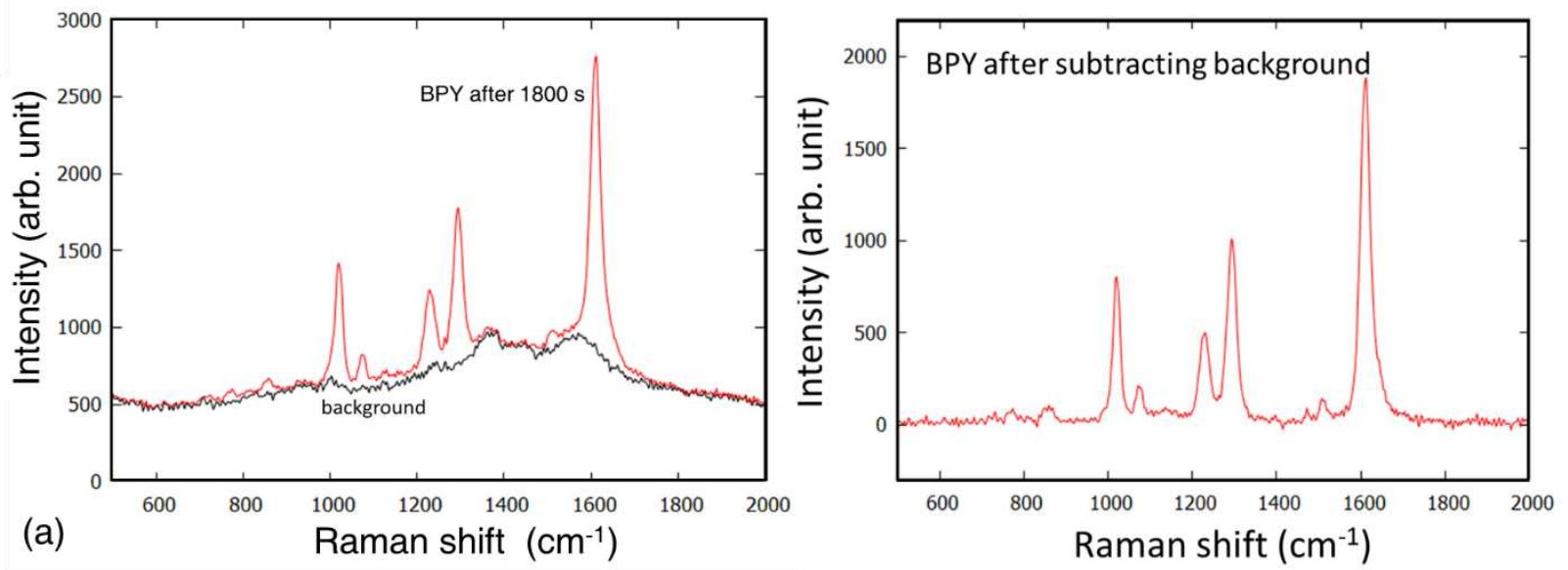

Fig. 2 (a) Background and SERS spectra of BPY and (b) difference spectra of BPY after $1800 \mathrm{~s}$.

154 BPY at 1000, 1200, 1265, and $1600 \mathrm{~cm}^{-1}$ were observed, which are attributed to the pyridine ring breathing, ring

155 deformation, $\mathrm{C}=\mathrm{C}$ in-plane ring mode, and $\mathrm{C}=\mathrm{C}$ stretching mode, respectively.(Lu et al. 1989) Fig. 2(b) shows the

156 difference spectrum obtained by subtracting the background spectrum from the SERS spectrum shown in Fig. 2(a).

157 Similar time-dependent difference spectra were also obtained for all the molecules by subtracting the background

158 spectra from the SERS spectrum. Fig. 3 shows the difference spectra for BPY, CV, Rh6G, and RB. The same

159 operation was performed for the other eight types of molecules. All the molecules diffused through the gel rapidly and

160 reached the Au nanoparticles in less than 60s, giving SERS peaks. For all the molecules, the SERS peak was observed

161 at the wavenumber unique to the molecule. In addition, Raman signal intensity increased continuously with time,

162 indicating the process of an increase in the concentration of the molecular solute arriving at the AuNPs was driven by

163 diffusion. BPE has the strongest peak intensity among the measured molecules, and SE, AO7, and AO12 exhibited

164 the weakest peak intensity even after increasing the exposure period by five times. Furthermore, because Acid Orange

1657 and Acid Orange 12 are structural isomers, their SERS spectra were identical.

\section{3.3 Diffusion dependent SERS Intensity}

167 Fig. 4 shows the highest peak area as a function of time. It is preferable to use the peak area because the peak widths 168 of SERS and ordinary Raman are different.(Pérez-Jiménez et al. 2020) In the SERS spectrum of every molecule, the 169 highest intensity peak was selected. The rise in the peak area can be considered directly proportional to the molecule 170 concentration arriving at the AuNP hotspot and was used for estimating the coefficient of diffusion. This curve can be 171 divided into three regions. In region I (red), probe molecules diffuse quickly within the gel and reach the AuNPs 
172 giving the first SERS signal. The time interval corresponds to when the probe molecule covers a distance equal to the 173 gel thickness. In region II (green), the peak intensity increases linearly and tends to saturate.
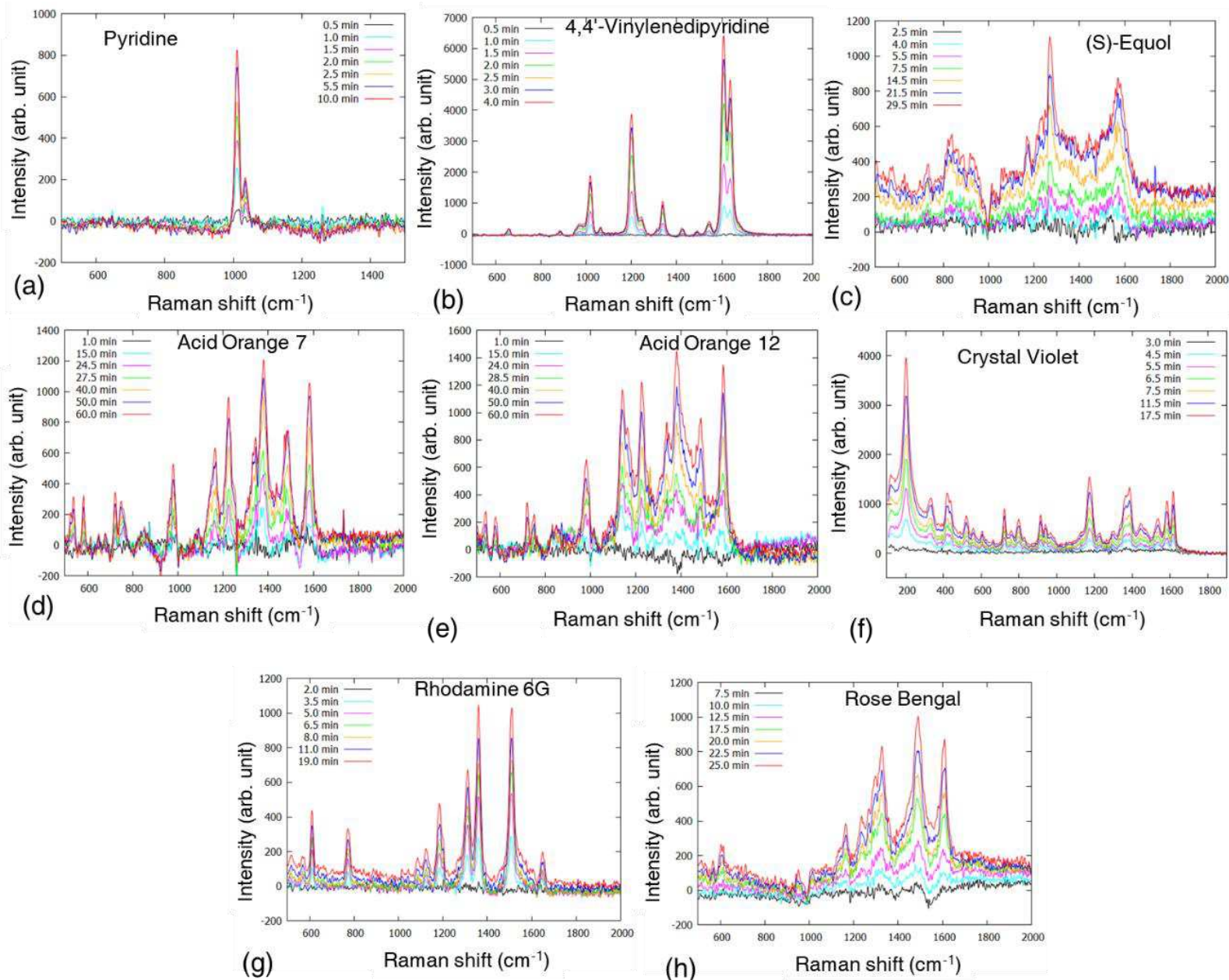

Fig. 3 Background subtracted SERS spectrum of (a) Pyridine, (b) 4,4-Vinylenedipyridine, (c) (S)-Equol, (d) Acid Orange 7, (e) Acid Orange 12, (f) Crystal Violet, (g) Rhodamine6G and (h) Rose Bengal, as a function of time.

174 The rise in peak intensity was found to be dependent upon the probe molecule. In other words, the transport

175 phenomenon of molecules in the gel differs depending on the molecular weight. Additionally, region I and region II's

176 width corresponds to the time of arrival and the time of saturation, respectively — was also found to depend on the

177 probe molecule. In region III (blue), the SERS intensity attained a plateau and was almost constant. This implies that

178 either the molecules have reached an equilibrium state with uniform concentration throughout the gel, or the number

179 of available SERS hotspots has been occupied.

180 The diffusion coefficient can be calculated by slicing the gel after the experiment and measuring the concentration of 181 solute as a function of time or by measuring the total amount of solute that penetrates the gel at a given time.(Lauffer 
184 corresponds to the diffusion-limited transport regime where our boundary conditions are valid (Eq. [5] and Eq. [6]),

185 see Figure 4.
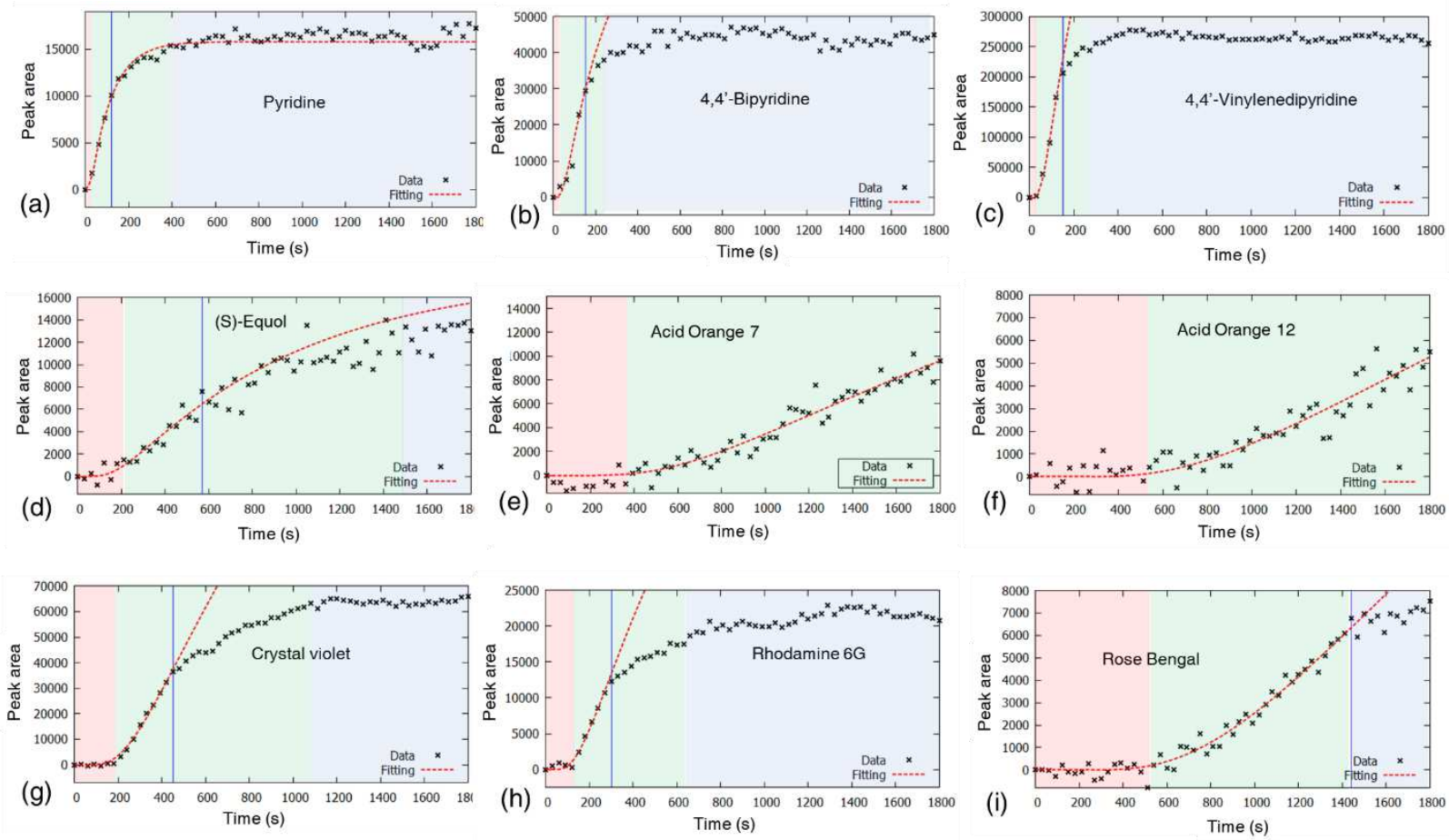

Fig. 4 Area of the highest peak as a function of time for (a) Pyridine, (b) 4,4-Bipyridine , (c) 4,4Vinylenedipyridine, (d) (S)-Equol, (e) Acid Orange 7, (f) Acid Orange 12, (g) Crystal Violet, (h) Rhodamine6G , and (i) Rose Bengal. The dotted red curve is the fitted curve using Eq. 7, and the black cross points are the experimental data. The blue line is the boundary condition for the fitted curve. Regions I, II, and III are colored red, green, and blue, respectively.

186 The graph shows a strong agreement between the experimental data and the fit. Since Acid Orange 7 and Acid Orange

18712 always showed a gradual increasing tendency across the measurement time $(3 \mathrm{~h})$, the fitting was done throughout the complete data set. Table 2 shows the diffusion coefficient $D$ of each molecule obtained by fitting.

Table 2. Diffusion coefficient $D$ of the nine molecules

\begin{tabular}{lc}
\hline Molecule name & $\begin{array}{l}\text { Diffusion coefficient } D \\
{\left[10^{-11} \mathrm{~m}^{2} / \mathrm{s}\right]}\end{array}$ \\
\hline Pyridine & 265 \\
\hline 4,4'-Bipyridine & 121 \\
\hline 4,4'-Vinylenedipyridine & 100 \\
\hline (S)-Equol & 30.9 \\
\hline Acid Orange 7 & 8.72 \\
\hline Acid Orange 12 & 6.68 \\
\hline Crystal Violet & 22.0 \\
\hline Rhodamine 6G & 36.8 \\
\hline Rose Bengal & 6.31 \\
\hline
\end{tabular}



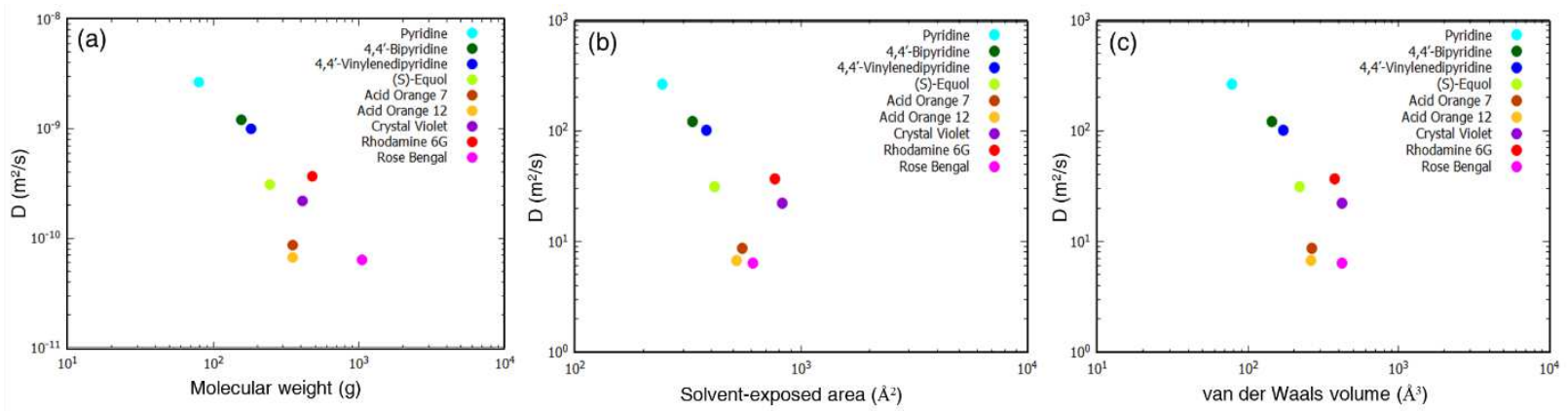

Fig. 5 Logarithmic plot of diffusion co-efficient as a function of (a) molecular weight, (b) solvent exposed area, (c) van der Waals volume.

191 We examined the diffusion coefficient as a function of molecular weight. Fig. 5(a) shows the logarithmic plot of $D$ as

192 a function of molecular weight. In general, the diffusion coefficient was found to decrease with the increase in the

193 molecular weight for all the probe molecules. Light molecules PY, BPY, and BPY were found to have the maximum,

194 and the heavier molecules like RB were found to have the lower diffusion coefficient in the decreasing order of their

195 molecular weight. This observation is consistent with the tendency based on the kinetic theory that molecules with

196 smaller and lighter molecular weights diffuse faster in the solvent. However, when examined closely, Rh6G has a

197 higher value of $D$ than $\mathrm{AO} 7, \mathrm{AO} 12$, or $\mathrm{CV}$; all three are lighter than Rh6G. Also, $\mathrm{AO} 7$ and $\mathrm{AO} 12$ have the value of

$198 \mathrm{D}$, which is almost like RB, which has almost thrice their molecular weight. Figures 5 (b) and 5 (c) show the diffusion

199 coefficient plotted as a function of vdW volume and SEA to understand this strange behavior better. The diffusion

200 coefficient $\mathrm{D}$ was found to have a similar tendency when plotted against vdW volume and SEA. This behavior is based

201 on the inhibitory effect of the gel substance that tends to inhibit movement as the molecular size increases and the

202 pathway length is extended. The trend was similar to the molecular weight for the lightest three molecules with the

203 highest $D$ value. Comparing CV and Rh6G, Rh6G has a higher molecular weight, while Crystal Violet has a higher

204 vdW volume and SEA than Rh6G, explaining its lower D value. Therefore, it can be considered that the vdW volume

205 and SEA are the major factors that affect the transport in the case of Rh6G. RB is the heaviest of all the test molecules

206 and has the lowest diffusion $D$ among the measured molecules.

207 Comparing the molecular size of $\mathrm{RB}$ with $\mathrm{CV}$, the $\mathrm{vdW}$ volume is about the same, and the solvent exposure area is

208 smaller than $\mathrm{CV}$. However, $\mathrm{CV}$ has a higher $D$. As a result, when compared to CV, the molecular weight has a more

209 significant effect on $D$ in the case of RB. The two types of molecules, AO7 and AO12, have a small diffusion 
210 coefficient $D$, even smaller than $\mathrm{CV}$ and Rh6G, even though they have smaller molecular weight values, vdW volume,

211 and SEA. This is assumed to be because of chemical factors such as the activity of functional groups increasing due

212 to the intricate molecular structure as the molecular weight and molecular size grow, affecting the molecule's diffusion.

213 AO7 and AO12 have a hydroxyl group that may have an inhibitory effect on the gel and can delay the diffusion of

214 molecules by binding the gel substance to the solvent. The three types of molecules with a large diffusion coefficient

$215 D$ do not have functional groups, and their molecular structures are relatively simple and like each other. Therefore, it

216 is considered that a few chemical factors contribute to the difference in molecular transport phenomena. (Johansson et

217 al. 1991, 2007) The difference in molecular diffusion was found to be based on the kinetic theory of molecules and

218 the inhibitory effect of gel substances, which means that the larger the molecular weight and size of the molecule, the

219 slower the diffusion.

\section{4. CONCLUSIONS}

221 In conclusion, we investigated the differences in transport processes of molecules of varying molecular weight as a

222 function of SERS intensity when dispersed across HEC gel. We investigated a model function that can characterize

223 this temporal change and derived the diffusion coefficient $\mathrm{D}$ for each molecule that matches the experimental

224 observations. The diffusion coefficient $\mathrm{D}$ was examined in connection with the molecular weight, vdW volume, and

225 solvent exposure area. This investigation agrees with prior observations based on the molecular motion concept that

226 diffusion slows as molecular weight and size increase and the inhibitory effect of the gel substance.

\section{Supplematary Information}

228 The molecular structure of probe molecules, the diffusion model in detail, and the SEM images of the SERS chip 229 utilized for this study can be found in the supplementary information.

\section{Acknowledgments}

231 Funding. This work was supported by JST COI under Grant Number JPMJCE1307.

\section{Declarations}

233 Conflict of interest. The authors have no conflicts of interest to declare. 
234 Human and animal rights. This article does not contain any studies with human participants or animals performed

235 by any of the authors.

\section{REFERENCES}

237 Amsden B (1998) Solute Diffusion within Hydrogels. Mechanisms and Models. Macromolecules 31:8382-8395

238 Askadskiı̌ AA (2003) Computational materials science of polymers. Cambridge Int Science Publishing

Bochenkov V, Baumberg J, Noginov M, et al (2015) Applications of plasmonics: general discussion. Faraday Discuss 178:435-466

241 Chen J, Huang M, Kong L, Lin M (2019a) Jellylike flexible nanocellulose SERS substrate for rapid in-situ noninvasive pesticide detection in fruits/vegetables. Carbohydr Polym 205:596-600

243 Chen Y-C, Chen J-J, Hsiao Y-J, et al (2021) Plasmonic gel films for time-lapse LSPR detection of hydrogen peroxide secreted from living cells. Sens Actuators B Chem 336:129725

Chen Y-C, Chen K-Y, Hsu K-H, Chen Y-F (2019b) Surface-enhanced Raman spectroscopy and localized surface plasmon resonance detection of hydrogen peroxide using plasmonic gels. In: Plasmonics in Biology and Medicine XVI. International Society for Optics and Photonics, p 108940R

El-Zahry MR, Lendl B (2018) Structure elucidation and degradation kinetic study of Ofloxacin using surface enhanced Raman spectroscopy. Spectrochim Acta A Mol Biomol Spectrosc 193:63-70

Gahlaut SK, Savargaonkar D, Sharan C, et al (2020) SERS platform for dengue diagnosis from clinical samples employing a hand held Raman spectrometer. Anal Chem 92:2527-2534

252 Hamelryck T (2005) An amino acid has two sides: a new 2D measure provides a different view of solvent exposure. Proteins 59:38-48

254 Hickey ME, He L (2021) SERS imaging analyses of bacteria cells among plant tissues. Talanta 225:122008

Hu X, Yang B, Wen X, et al (2021) One-pot synthesis of a three-dimensional Au-decorated cellulose nanocomposite as a surface-enhanced Raman scattering sensor for selective detection and in situ monitoring. ACS Sustain Chem Eng. https://doi.org/10.1021/acssuschemeng.0c09296

Innocenzi P, Malfatti L (2019) Mesoporous materials as platforms for surface-enhanced Raman scattering. Trends Analyt Chem 114:233-241

Jain RK (1987) Transport of molecules in the tumor interstitium: a review. Cancer Res 47:3039-3051

Johansson L, Elvingson C, Skantze U, Löfroth JE (2007) Diffusion and interaction in gels and solutions. In: Progress in Colloid \& Polymer Science. Steinkopff, Darmstadt, pp 25-29

Johansson L, Skantze U, Loefroth JE (1991) Diffusion and interaction in gels and solutions. 2. Experimental results on the obstruction effect. Macromolecules 24:6019-6023

Joo S-W (2004) Surface-enhanced Raman scattering of 4,4'-bipyridine on gold nanoparticle surfaces. Vib Spectrosc 34:269-272

Joseph MM, Narayanan N, Nair JB, et al (2018) Exploring the margins of SERS in practical domain: An emerging diagnostic modality for modern biomedical applications. Biomaterials 181:140-181 
Kim DJ, Yoon J, Kim D-H, et al (2020) Plasmonic microgels for Raman-based molecular detection created by simultaneous photoreduction and photocross-linking. ACS Appl Mater Interfaces 12:48188-48197

Kumar S, Doi Y, Namura K, Suzuki M (2020a) Plasmonic nanoslit arrays fabricated by serial bideposition: Optical and surface-enhanced Raman scattering study. ACS Applied Bio Materials

Kumar S, Goel P, Singh DP (2014) Fabrication of superhydrophobic silver nanorods array substrate using glancing angle deposition. AIP Conf Proc

Kumar S, Kanagawa M, Namura K, et al (2020b) Multilayer thin-film flake dispersion gel for surface-enhanced Raman spectroscopy. Appl Nanosci. https://doi.org/10.1007/s13204-020-01562-0

277 Kumar S, Kumar P, Das A, Shakher Pathak C (2020c) Surface-Enhanced Raman Scattering: Introduction and Applications. In: Recent Advances in Nanophotonics - Fundamentals and Applications. IntechOpen

Kumar S, Lodhi DK, Goel P, et al (2015) A facile method for fabrication of buckled PDMS silver nanorod arrays as active 3D SERS cages for bacterial sensing. Chem Commun 51:12411-12414

Kumar S, Namura K, Kumaki D, et al (2020d) Highly reproducible, large scale inkjet-printed Ag nanoparticles-ink SERS substrate. Results in Materials 8:100139

Kumar S, Namura K, Suzuki M (2019) Proposal for a gel-based SERS sensor. In: Plasmonics in Biology and Medicine XVI. International Society for Optics and Photonics, p 1089414

Kumar S, Tokunaga K, Namura K, et al (2020e) Experimental evidence of a twofold electromagnetic enhancement mechanism of surface-enhanced Raman scattering. J Phys Chem C Nanomater Interfaces 124:21215-21222

287 Lauffer MA (1961) Theory of diffusion in gels. Biophys J 1:205-213

Le Ru EC, Etchegoin PG (2009) SERS enhancement factors and related topics. In: Principles of Surface-Enhanced Raman Spectroscopy. Elsevier, pp 185-264

Lock JY, Carlson TL, Carrier RL (2018) Mucus models to evaluate the diffusion of drugs and particles. Adv Drug Deliv Rev 124:34-49

292 Lu T, Cotton TM, Birke RL, Lombardi JR (1989) Raman and surface-enhanced Raman spectroscopy of the three

Mayr J, Saldías C, Díaz Díaz D (2018) Release of small bioactive molecules from physical gels. Chem Soc Rev 47:1484-1515

McNay G, Eustace D, Smith WE, et al (2011) Surface-enhanced Raman scattering (SERS) and surface-enhanced resonance Raman scattering (SERRS): a review of applications. Appl Spectrosc 65:825-837

298 Muhr AH, Blanshard JMV (1982) Diffusion in gels. Polymer 23:1012-1026

Ogundare SA, van Zyl WE (2019) A review of cellulose-based substrates for SERS: fundamentals, design principles, applications. Cellulose 26:6489-6528

301 Pérez-Jiménez AI, Lyu D, Lu Z, et al (2020) Surface-enhanced Raman spectroscopy: benefits, trade-offs and future developments. Chem Sci 11:4563-4577

303 Premasiri WR, Chen Y, Fore J, et al (2018) SERS Biomedical Applications: Diagnostics, Forensics, and Metabolomics.

$304 \quad$ In: Frontiers and Advances in Molecular Spectroscopy. Elsevier, pp 327-367 
Rajput A, Kumar S, Singh JP (2017) Vertically standing nanoporous Al-Ag zig-zag silver nanorod arrays for highly active SERS substrates. Analyst 142:3959-3966

307 Salemmilani R, Mirsafavi RY, Fountain AW, et al (2019) Quantitative surface-enhanced Raman spectroscopy chemical analysis using citrate as an in situ calibrant. Analyst 144:1818-1824

Samprovalaki K, Robbins PT, Fryer PJ (2012) Investigation of the diffusion of dyes in agar gels. J Food Eng 111:537545

311 Sandrin D, Wagner D, Sitta CE, et al (2016) Diffusion of macromolecules in a polymer hydrogel: from microscopic 312 to macroscopic scales. Phys Chem Chem Phys 18:12860-12876

313 Schlucker S (ed) (2013) Surface enhanced Raman spectroscopy, 1st edn. Wiley-VCH Verlag, Weinheim, Germany

Segawa H, Fukuoka T, Itoh T, et al (2019a) Rapid detection of synthetic cannabinoids in herbal highs using surfaceenhanced Raman scattering produced by gold nanoparticle co-aggregation in a wet system. Analyst 144:6928-6935

Segawa H, Fukuoka T, Itoh T, et al (2019b) Rapid detection of hypnotics using surface-enhanced Raman scattering based on gold nanoparticle co-aggregation in a wet system. Analyst 144:2158-2165

Sharma B, Frontiera RR, Henry A-I, et al (2012) SERS: Materials, applications, and the future. Mater Today (Kidlington) 15:16-25

Singh N, Kumar P, Riaz U (2019) Applications of near infrared and surface enhanced Raman scattering techniques in tumor imaging: A short review. Spectrochim Acta A Mol Biomol Spectrosc 222:117279

323 Suzuki M, Maekita W, Kishimoto K, et al (2005) Direct formation of arrays of prolate Ag nanoparticles by dynamic

Suzuki M, Maekita W, Wada Y, et al (2006) In-line aligned and bottom-up Ag nanorods for surface-enhanced Raman spectroscopy. Appl Phys Lett 88:203121

Suzuki M, Nakajima K, Kimura K, et al (2007) Au nanorod arrays tailored for surface-enhanced Raman spectroscopy. Anal Sci 23:829-833

329 Tokita M (2016) Transport Phenomena in Gel. Gels 2:. https://doi.org/10.3390/gels2020017

Wang YY, Jiang J, Yin J, et al (2019) Determination of concentration of adsorbed molecules by Raman spectroscopy and optical imaging. J Appl Phys 125:244305

Wu L-A, Chen Y-C, Pai W-C, et al (2020) Plasmonic nanoparticles in agarose gel and filter paper-integrated microfluidic devices for SERS detection of molecules. In: Plasmonics in Biology and Medicine XVII. International Society for Optics and Photonics, p 1125702

Yadav S, Senapati S, Desai D, et al (2021) Portable and sensitive Ag nanorods based SERS platform for rapid HIV-1 detection and tropism determination. Colloids Surf B Biointerfaces 198:111477

337 Yu WW, White IM (2013) Chromatographic separation and detection of target analytes from complex samples using inkjet printed SERS substrates. Analyst 138:3679-3686

Yu X, Li W, Liang O, et al (2020) Molecular orientation and specificity in the identification of biomolecules via surface enhanced Raman spectroscopy. Anal Biochem 599:113709 
343

344 


\section{Supplementary Files}

This is a list of supplementary files associated with this preprint. Click to download.

- SERSDiffusionESI20210602v1.pdf 